\title{
Multiple Models Adaptive Switching Control Based on Feasible Controller Set
}

\author{
Jian Wang and Chunbao Huo \\ The Information Engineering department \\ Liaoning Institute of Technology \\ Jinzhou, China \\ Wjth100@sina.com
}

\author{
Meng Zhao \\ The Institute of Mechanics \\ Chinese Academy of Science \\ Beijing, China
}

\begin{abstract}
In this paper a new systematic switching control approach to adaptive stabilization of parameters uncertain linear systems is presented. One feature of this approach based on feasible controller set is its controller falsification capability, which is manifested as the rapid convergence of the switching controller, another feature is its capability of improving the closeloop transient response and reducing computation burden. In addition, the potential advantages of the presented approach include the applicability to both continuous and discrete uncertainty system and the simplicity of the stability analysis.
\end{abstract}

Keywords- Feasible controller set; Multiple models; Switching control; Uncertain system

\section{INTRODUCTION}

In the traditional design of controllers for plant uncertainty, the general approaches often adapted are adaptive control and robust control. As far as adaptive control is concerned, if sudden change in plant dynamic happens due to operating environment, component failure or extemal influence, the transient response may be poor and system is even unstable. As for robust control $\left(\mathrm{H}_{2}, \mathrm{H}_{\infty}\right.$ and the like), they can only deal with the model of which uncertainties are "sufficiently small".

To cope with "large model uncertainties" and improve transient response, switching control is developed in recent years [1]-[9]. There are two kinds of switching control methods: (1) Evaluating every candidate controller's performance by applying it to the process in a predetermined sequence [1]-[4]; (2) orchestrating feedback controllers into the process of switching from a precomputed finite (continuum) set of fixed controllers based on certain online estimation [5]-[8]. The first method has the advantage of light computation burden, but the controller search may converge very slowly resulting in excessive transients which renders the system unstable in a practical sense; the second method can improve the system transient response, however the computation burden of this method is heavy when more models are considered and several issue still remain unresolved, these include the controller being nonconvergent and the proofs of stability being too complicated [5].

In this paper, a new class of adaptive switching mechanisms is presented to overcome the drawbacks mentioned above. This approach is based on the feasible controller set which incorporates simultaneous falsification of a number of controllers and therefore, improves controller converge rate and reduces online estimation computation burden. The potential advantages of this approach include the finite convergence for switching, the simplicity of the stability analysis and the applicability to both continuous and discrete system.

\section{PROBLEM FORMULATION AND B ASIC LEMMA}

It is assumed that the uncertain plant to be controlled may be described by a model $\mathrm{P}_{\mathrm{m}}$ contained in a finite set $\mathrm{P}$ of parameter uncertain continuous linear system models, and assumed that a finite set of robust controllers $\left\{\Pi_{i}\right\}$, $i=1,2, \ldots \ldots s, s \in N$, has been found so that for each plant $P_{i} \in$ $P, i=1,2, \ldots \ldots . s$, there exists one controller $\Pi_{i}, i=1,2, \ldots \ldots s$, so that the resulting closed-loop system $\left\{\mathrm{P}_{\mathrm{i}}, \Pi_{i}\right\}$ is stable, a switching control algorithm is proposed in this paper to select the real controller out of controller set $\left\{\Pi_{i}\right\}$ with the property of stability of closed-loop system.

Where

$$
\begin{gathered}
\mathrm{P}=\bigcup_{\mathrm{i}=1}^{s} \mathrm{P}_{\mathrm{i}} \\
\mathrm{P}_{\mathrm{i}}:\left\{\begin{array}{l}
\dot{\mathrm{x}}=\mathrm{A}_{\mathrm{i}} \mathrm{x}+\mathrm{B}_{\mathrm{i} 1} \mathrm{u}+\mathrm{B}_{\mathrm{i} 2} \omega \\
\mathrm{y}=\mathrm{C}_{\mathrm{i} 1} \mathrm{x}+\mathrm{D}_{\mathrm{i} 12} \omega \\
\mathrm{z}=\mathrm{C}_{\mathrm{i} 2} \mathrm{x}+\mathrm{D}_{\mathrm{i} 21} \mathrm{u}+\mathrm{D}_{\mathrm{i} 22} \omega
\end{array}\right. \\
\mathrm{A}_{\mathrm{i}}=\mathrm{A}_{\mathrm{ni}}+\Delta \mathrm{A}_{\mathrm{i}} \quad \mathrm{i}=1,2, \ldots \ldots \mathrm{s}
\end{gathered}
$$

Where $A_{n i}$ is the constant matrix and $\Delta A_{i}$ is uncertain parameter. The others system parameter matrices have the same form as $A_{1}$ with appropriate dimension and all uncertain parameters $\left(\Delta \mathrm{A}_{\mathrm{i}}, \Delta \mathrm{B}_{\mathrm{i1}}, \Delta \mathrm{B}_{\mathrm{i} 2}, \Delta \mathrm{C}_{\mathrm{i1}}, \Delta \mathrm{C}_{\mathrm{i} 2}, \Delta \mathrm{D}_{\mathrm{i} 12}, \Delta \mathrm{D}_{\mathrm{i} 21}\right.$,

$\Delta D_{i 22}$ ) satisfy norm-bounded parameter uncertainties.

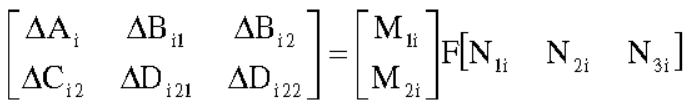

$$
\begin{aligned}
& {\left[\begin{array}{ll}
\Delta \mathrm{C}_{\mathrm{i} 1} & \Delta \mathrm{D}_{\mathrm{i} 12}
\end{array}\right]=\mathrm{M}_{3 \mathrm{i}} \mathrm{F}\left[\mathrm{N}_{1 \mathrm{i}} \quad \mathrm{N}_{3 \mathrm{i}}\right] \quad \mathrm{i}=1,2, \ldots . \mathrm{s}}
\end{aligned}
$$

Where $F$ is an unknown real matrix satisfying

$$
\mathrm{FF}^{\mathrm{T}} \leq \mathrm{I}
$$

$\mathrm{M}_{1 \mathrm{i}}, \mathrm{M}_{2 \mathrm{i}}, \mathrm{M}_{3 \mathrm{i}}, \mathrm{N}_{1 \mathrm{i}}, \mathrm{N}_{2 \mathrm{i}}, \mathrm{N}_{3 \mathrm{i}}$ are the known matrices with appropriate dimension. 


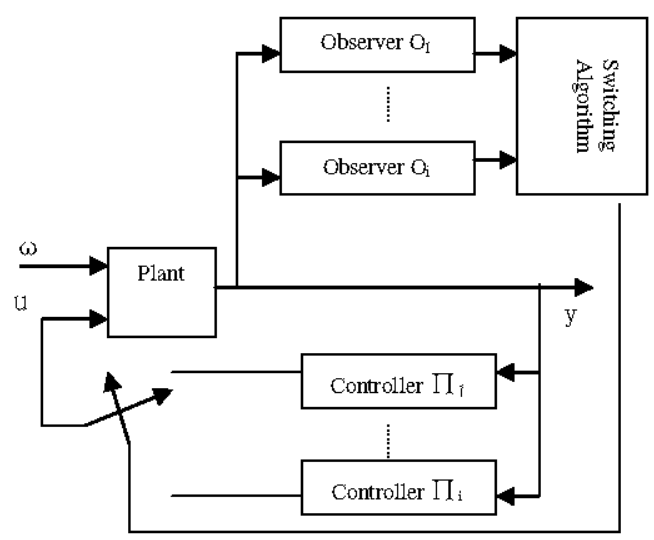

Figure 1 The schematic block diagram of switching control

A robust $H_{\infty}$ controller $\Pi_{i}$ is designed for each models $P_{i}$ so that the resulting closed-loop system $\left\{\mathrm{P}_{\mathrm{i}}, \Pi_{\mathrm{i}}\right\}$ is stable and all controllers compose controller set $\Pi$,

$$
\begin{gathered}
\Pi=\bigcup_{i=1}^{s} \Pi_{\dot{1}} \\
\Pi_{\dot{1}}:\left\{\begin{array}{l}
\dot{\varepsilon}=A_{i}^{\Pi} \varepsilon+B_{i}^{\Pi} y \\
u=C_{i}^{\Pi} \varepsilon \\
i=1,2, \ldots \ldots s
\end{array}\right.
\end{gathered}
$$

Where $\varepsilon$ is controller state.

A robust $\mathrm{H}_{\infty}$ observer $\mathrm{O}_{1}$ is designed for each close-loop system $\left\{\mathrm{P}_{\mathrm{i}}, \Pi_{\mathrm{i}}\right\}$ ( the model $\mathrm{P}_{\mathrm{i}}$ and its controller $\Pi_{\mathrm{i}}$ ), $\mathrm{i}=1,2, \cdots \mathrm{s}$.

$$
\mathrm{O}_{\mathrm{i}}:\left\{\begin{array}{c}
\dot{\eta}=A_{i}^{0} \eta+B_{i}^{0} y \\
y_{\text {io }}=C_{i}^{0} \eta+D_{i}^{0} y \\
i=1,2, \ldots \ldots s
\end{array}\right.
$$

Where $\eta$ is observer state, $y_{i 0}$ is observer output.

Remark 2.1: The robust $\mathrm{H}_{\infty}$ observer $\mathrm{O}_{1}$ can not be designed for an unstable parameters uncertain system as (1), it is designed only for the stable system $\left\{\mathrm{P}_{1}, \Pi_{i}\right\}$.

Assume that $\mathrm{P}_{\mathrm{m}} \in \mathrm{P}, 1 \leq \mathrm{m} \leq \mathrm{s}$ is the real plant model.

$$
P_{m}:\left\{\begin{array}{l}
\dot{x}=A_{m} x+B_{m 1} u+B_{m 2} \omega \\
y=C_{m 1} x+D_{m 12} \omega \\
z=C_{m 2} x+D_{m 21} u+D_{m 22} \omega
\end{array}\right.
$$

The close-loop system that results on applying controller $\Pi_{\dot{1}}$ $(\mathrm{i}=1,2, \ldots \ldots . . \mathrm{s})$ to $\mathrm{P}_{\mathrm{m}}$ is $\Pi_{\mathrm{i}} \mathrm{P}_{\mathrm{m}}$,

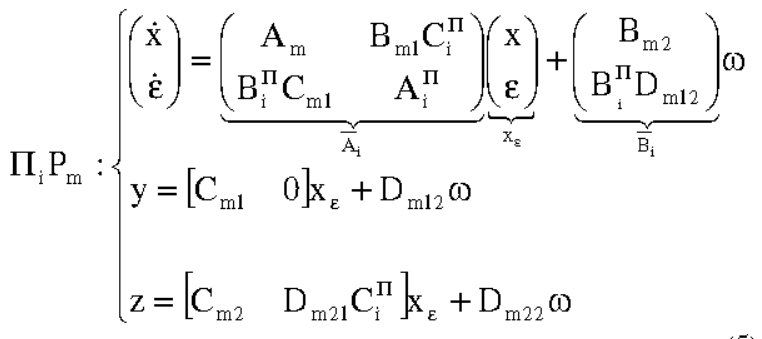

Combining (3) with (5), we obtain the following augmented system $\left\{\prod_{i} P_{n n} O_{i}\right\}, i=\{1,2, \ldots \ldots s\}$

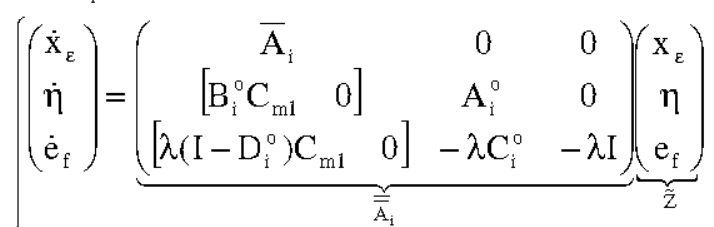

$$
\begin{aligned}
& +\underbrace{\left(\begin{array}{c}
\overline{\mathrm{B}}_{\mathrm{i}} \\
\mathrm{B}_{\mathrm{i}}^{\circ} \mathrm{D}_{\mathrm{m} 12} \\
\lambda\left(\mathrm{I}-\mathrm{D}_{\mathrm{i}}^{\circ}\right) \mathrm{D}_{\mathrm{m} 12}
\end{array}\right)}_{\overline{\overline{\mathrm{B}}_{\mathrm{i}}}} \omega
\end{aligned}
$$

Where $e_{f}$ is the filtered observer error $e_{i}=y-y_{i 0}$ given by

$$
\begin{aligned}
& \dot{\mathrm{e}}_{\mathrm{f}}(\mathrm{t})=-\lambda \mathrm{e}_{\mathrm{f}}(\mathrm{t})+\lambda \mathrm{e}_{\mathrm{i}}(\mathrm{t}) \\
& \lambda>0
\end{aligned}
$$

When the controller $\Pi_{i}$ is applied to $P_{m}$, the filtered observer error $\mathrm{e}_{\mathrm{j}}=\mathrm{y}-\mathrm{y}_{\mathrm{j} 0}$ of each observer $\mathrm{O}_{\mathrm{j}}$ is obtained by augmented system $\left\{\Pi_{i} P_{m} O_{j}\right\}, i, j=1,2, \ldots \ldots . s$.

$$
\begin{aligned}
& \dot{\mathrm{e}}_{\mathrm{fj}}(\mathrm{t})=-\lambda \mathrm{e}_{\mathrm{fj}}(\mathrm{t})+\lambda \mathrm{e}_{\mathrm{j}}(\mathrm{t})
\end{aligned}
$$

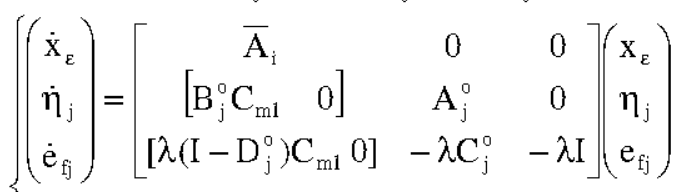

$$
\begin{aligned}
& +\left[\begin{array}{c}
\bar{B}_{i} \\
B_{j}^{0} D_{m 12} \\
\lambda\left(I-D_{j}^{\circ}\right) D_{m 12}
\end{array}\right] \omega
\end{aligned}
$$

Throughout this paper, the $\infty$-norm of $x=\left[x_{1}, \ldots \ldots, x_{n}\right] \in$ $\mathrm{R}^{\mathrm{m}}$ and the induced $\infty$-norm of $\mathrm{X} \in \mathrm{R}^{\mathrm{m} \times \mathrm{n}}$ is denoted respectively as

$\|x\|:=\max _{i \in\{1,2, \ldots m\}}\left|x_{i}\right|$

$\|X\|:=\max _{\mathrm{i} \in\{1,2, \ldots \mathrm{m}\}} \sum_{\mathrm{j}=1}^{\mathrm{n}}\left|\mathrm{X}_{\mathrm{ij}}\right|$

As well, the $\mathrm{L}_{\infty}$ norm of a function $\mathrm{f}: \mathrm{R}^{+} \cup\{0\} \rightarrow \mathrm{R}^{\mathrm{n}}$

is denoted as $\|f\|:=$ ess sup $\|f(t)\|$ 
lemma2.1: if $\overline{\bar{A}}_{i}$ of (6) is Hurwitz matrix, and $\omega(t)$ is piecewise continuous bounded signal having $\mathrm{L}_{\infty}$ norms of $\bar{\omega}$, then there exist constants $\xi_{1}>0, \xi_{2}>0$, such that for all $\mathrm{t}>0$,

Proof: See [1] lemma 1 .

$$
\|\tilde{z}(\mathrm{t})\| \leq \xi_{1}\|\widetilde{z}(0)\|+\xi_{2}
$$

\section{Switching Control Based on Feasible CONTROLler SET}

The presented switching control algorithm in this paper is based on the feasible controller set which incorporates simultaneous falsification of a number of controllers, therefore improves controller converge rate and reduces computation burden. The algorithm will determine the controller switching time and select the correct controller from the feasible controller set for an unknown uncertain plant $\mathrm{P}_{\mathrm{m}}$. The schematic block diagram of switching control is shown as Fig 1.

Definition 3.1: A function $f: N \rightarrow R$ is said to bounding function if it is strictly increasing and if for certain constants $\mathrm{c}_{0}>0, \mathrm{c}_{1}>0$,

$$
\mathrm{f}(\mathrm{p}) /\left(\mathrm{c}_{0}+\mathrm{c}_{1} \sum_{\mathrm{i}=1}^{\mathrm{p}-1} \mathrm{f}(\mathrm{i})\right)>1
$$

Definition 3.2: A set $F\left(t_{k}\right)=\left\{\Pi_{j}\right\}$ is said to be feasible controller set at the kth switching time $t_{k}$ if when the controller $\Pi_{i}$ is applied to $P_{m}$, the augmented system $\left\{\Pi_{\mathrm{i}} \mathrm{P}_{\mathrm{m}} \mathrm{O}_{\mathrm{j}}\right\}$ meets the following inequality at $\mathrm{t}_{\mathrm{k}}$,

$$
\left\|\eta_{j}\left(t_{k}\right), e_{f_{j}}\left(t_{k}\right)\right\|<b_{0}+b_{1} \sum_{i=1}^{k-1} f(i)
$$

for certain constants $b_{0}>0$ and $b_{1}>0$, i.e:

$\mathrm{F}\left(\mathrm{t}_{\mathrm{k}}\right)=\left\{\Pi_{\mathrm{j}} \mid\right.$ the augmented system $\left\{\Pi_{\mathrm{i}} \mathrm{P}_{\mathrm{m}} \mathrm{O}_{\mathrm{j}}\right\}$ meets $(11)$ according to $(8)\}$.

Remark 3.1: According to the definition of $F\left(t_{k}\right)$, if the state and the error of the observer $\mathrm{O}_{\mathrm{j}}$ meet (11), the corresponding controller $\Pi_{j}$ of $O_{j}$ belongs to $F\left(t_{k}\right)$. That a controller $\Pi_{j}$ is said to be feasible controller means that it is "possible" for $\Pi_{j}$ to make the system stable, in other word, if a observer does not meet (11), its corresponding controller must not be real controller. See the property (1) of theorem 4.1.

Switching control algorithm for linear continuous systems:

\section{Algorithm 3.1:}

1). At $t=$ initial time $t_{1}$, let the feasible controller set $F\left(t_{1}\right)=\{$ $\left.\Pi_{1}, \Pi_{2}, \ldots \ldots \Pi_{s}\right\}$.

2). For $t>=t_{1}$, apply any controller (for example $\Pi_{j}$ ) of the feasible controller set $F\left(t_{k-1}\right)$ to plant at $t_{k-1} \quad(k>=2)$ and determine the switching time $\mathrm{t}_{\mathrm{k}}$ by $\mathrm{t}_{\mathrm{k}}:=\left\{\begin{array}{l}\min \left\{\mathrm{t} \mid \mathrm{t}>\mathrm{t}_{\mathrm{k}-1},\left\|\left[\varepsilon(\mathrm{t})^{\mathrm{T}}, \eta(\mathrm{t})^{\mathrm{T}}, \mathrm{e}_{\mathrm{f}}(\mathrm{t})^{\mathrm{T}}\right]^{\mathrm{T}}\right\|=\mathrm{f}(\mathrm{k}-1)\right\} \\ \text { if this minimumexist } \\ \infty \quad \text { otherwise }\end{array}\right.$ according to (6).

3). If $t_{k}=\infty$, end. Otherwise modify $F\left(t_{k-1}\right)$ :

$$
\mathrm{F}\left(\mathrm{t}_{\mathrm{k}-1}\right)=\mathrm{F}\left(\mathrm{t}_{\mathrm{k}-1}\right) / \Pi_{\mathrm{j}}
$$

4). Determine the feasible controller set $F\left(t_{k}\right)$ at $t_{k}$ in $F\left(t_{k-1}\right)$

$$
\begin{aligned}
F\left(t_{k}\right) & =\left\{\Pi_{n} \mid \Pi_{n} \in F\left(t_{k-1}\right)\right. \\
\& & \left.\left\|\left[\eta_{n}\left(t_{k}\right)^{T}, e_{f n}\left(t_{k}\right)^{T}\right]^{T}\right\|<b_{0}+b_{1} \sum_{i=1}^{k-1} f(i)\right\}
\end{aligned}
$$

according to (8).

5). $\mathrm{k}=\mathrm{k}+1$

6). Return 2).

Remark 3.2: The feasible controller set method is partly similar to the Localization method [9] which apply to only discrete system, the feasible controller set method, however, can apply to not only discrete system, but also continuous system.

\section{MaIN Results}

\section{Assumption 4.1:}

$$
\begin{aligned}
& \text { (1) }\left\|\left[\varepsilon\left(t_{1}\right)^{\mathrm{T}}, \eta\left(\mathrm{t}_{1}\right)^{\mathrm{T}}, \mathrm{e}_{\mathrm{f}}\left(\mathrm{t}_{1}\right)^{\mathrm{T}}\right]^{\mathrm{T}}\right\|<\mathrm{f}(1) \\
& \text { (2) }\left\|\left[\eta_{\mathrm{i}}\left(\mathrm{t}_{1}\right)^{\mathrm{T}}, \mathrm{e}_{\mathrm{fi}}\left(\mathrm{t}_{1}\right)^{\mathrm{T}}\right]^{\mathrm{T}}\right\|<\mathrm{b}_{0}+\mathrm{b}_{1} \mathrm{f}(1)
\end{aligned}
$$$$
\mathbf{i}=1,2, \ldots . \mathrm{s}
$$

Theorem4.1. Consider a plant contained in $\mathrm{P}$ to which algorithm 3.1 is applied at time $\mathrm{t}=\mathrm{t}_{1}$ and assumption 4.1 holds, then for bounded piecewise continuous signal $\omega$, bounding function $f$ and certain positive constants $b_{0}, b_{1}, c_{0}, c_{1}$, the closeloop system has following properties that:

1).the state of the real observer $O_{m}$ and the filtered real observer error satisfy:

$$
\left\|\left[\eta_{\mathrm{m}}\left(\mathrm{t}_{\mathrm{k}}\right)^{\mathrm{T}}, \mathrm{e}_{\mathrm{fm}}\left(\mathrm{t}_{\mathrm{k}}\right)^{\mathrm{T}}\right]^{\mathrm{T}}\right\|<\mathrm{b}_{0}+\mathrm{b}_{1} \sum_{\mathrm{i}=1}^{\mathrm{k}-1} \mathrm{f}(\mathrm{i})
$$

at $t_{k}$ for suitable constants $b_{0}>0, b_{1}>0$.

2).there exist a finite time $t_{j}>=t_{1}$ and constant controller $\Pi_{i}$ such that for $t>=t_{j}, \Pi(j)=\Pi_{i}, i=\{1,2, \ldots \ldots s\}, \Pi(j)$ is the controller applied to the system at $\mathrm{t}_{\mathrm{j}}$.

Proof: The proof is too long, so omitted, refer to [1],[2]

The theorem manifests that after $t_{j}$, the controller is not switched and the real controller is found to make system stable.

Switching controller in this paper has following advantages:

1). Since (11) is the necessary condition that the real observer should meet, it is used as the distinguishing condition of the feasible controllers: If a observer does not meet (11), its corresponding controller must not be the real controller, so the feasible controller set contains the real controller. Since the feasible controller set $F\left(t_{k}\right)$ at $t_{k}$ is determined in $F\left(t_{k-1}\right)$ by algorithm 3.1, the relationship:

$$
\mathrm{F}\left(\mathrm{t}_{1}\right) \supseteq \mathrm{F}\left(\mathrm{t}_{2}\right) \supseteq \ldots \ldots \mathrm{F}\left(\mathrm{t}_{\mathrm{k}-1}\right) \supseteq \mathrm{F}\left(\mathrm{t}_{\mathrm{k}}\right) \supseteq \ldots \ldots
$$




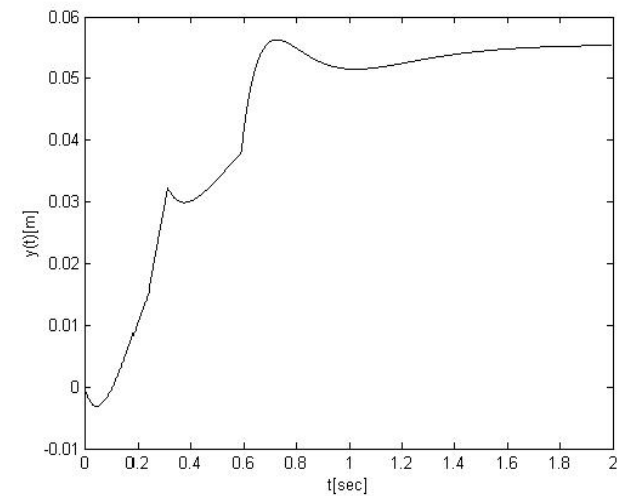

Figure2.The output response of system with feasible controller set method

holds, the scope of the feasible controller set in which the real controller exists can be reduced after the controller is switched each time, i.e. the number of the controllers and observers in Fig1 can be reduced after each switching, so controller can converge fast and online estimation computation burden can be reduced.

2). The switching control method in this paper is very simple and the distinguishing condition is the function of the switching number, it is therefore realistic to implement from a practical point of view.

3).Although the switching control algorithm is designed for continuous system. It (and theorem 4.1) can be generalized to discrete system routinely.

\section{Simulation Results}

In this section, the switching control for the following family of 6 plant models is considered. For simplicity and no loss of generation, 6 models adopted for simulation is the certain models. Due to space limitation of the paper, 6 controllers of the models $\mathrm{P}_{\mathrm{i}}(\mathrm{i}=1,2, \ldots 6)$ and 6 observers of the closed-loop systems $\left\{\mathrm{P}_{1}, \Pi_{1}\right\}, \mathrm{i}=1,2, \ldots 6$, are not given. Each model $P_{i}$ has the form of

$$
P_{i}:\left\{\begin{array}{l}
\dot{x}=A_{i 1} x+B_{i 1} u+B_{i 2} \omega \\
y=C_{i 1} x \\
z=C_{i 2} x
\end{array}\right.
$$

Where

$\mathrm{A}_{1}=[3,-17 ;-67,-3] ; \mathrm{B}_{11}=[1 ;-4.3] ; \mathrm{B}_{12}=[0.1 ; 0.1]$;

$\mathrm{C}_{11}=[5,7] ; \mathrm{C}_{12}=\mathrm{C}_{11}$;

$\mathrm{A}_{2}=[-75,7.25 ;-2.25,-8.25] ; \mathrm{B}_{21}=[0.1 ; 0.4] ; \mathrm{B}_{22}=[0.1 ; 0.1]$;

$\mathrm{C}_{21}=[-1.2,4.1] ; \mathrm{C}_{22}=\mathrm{C}_{21}$;

$\mathrm{A}_{3}=[14,21.1 ;-46.5,-25.4] ; \mathrm{B}_{31}=[-0.18 ; 0.33] ; \mathrm{B}_{32}=[0.1 ; 0.1]$;

$\mathrm{C}_{31}=[-0.45,7] ; \mathrm{C}_{32}=\mathrm{C}_{31}$;

$\mathrm{A}_{4}=[2.8,-18 ;-50,-2.4] ; \mathrm{B}_{41}=[1 ;-3.3] ; \mathrm{B}_{42}=[0.1 ; 0.1]$;

$\mathrm{C}_{41}=[4.5,7] ; \mathrm{C}_{42}=\mathrm{C}_{41}$;

$\mathrm{A}_{5}=[3.4,-24 ;-64,-3] ; \mathrm{B}_{51}=[1 ;-4] ; \mathrm{B}_{52}=[0.2 ; 0.1]$;

$\mathrm{C}_{51}=[0.5,3] ; \mathrm{C}_{52}=\mathrm{C}_{51}$

$\mathrm{A}_{6}=[-1.9,10.9 ;-1.3,-12.2] ; \mathrm{B}_{61}=[0 ; 0.1] ; \mathrm{B}_{62}=[0.1 ; 0.1]$;

$\mathrm{C}_{61}=[0.2,-0.5] ; \mathrm{C}_{62}=\mathrm{C}_{61}$;

The bounding function $f(k)=\exp (\mathrm{k}) / 200, b_{0}=0.015$, $b_{1}=1.08, \omega(t)=5$. Assume that $P_{6}$ is the real plant.

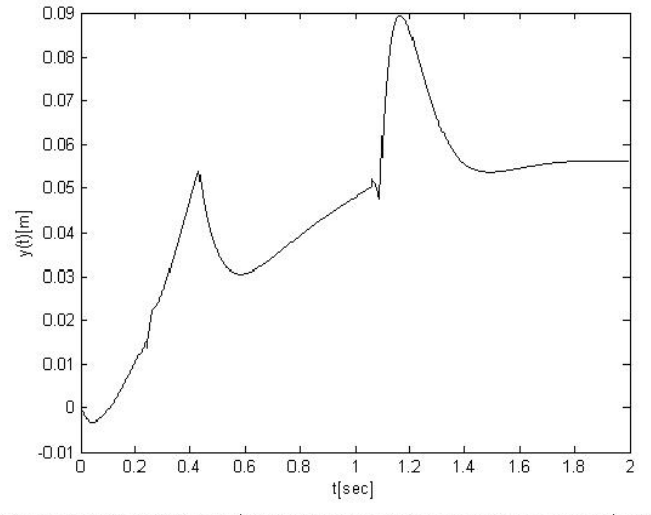

Figure3.The output responds of switching system that does not adopt feasible controller set method (no step 4 in algorithm 3.1)

The output responds of the switching system by algorithm 3.1(feasible controller set method) is shown in Fig2, the output responds of the switching system that does not adopted the feasible controller set method (no step 4 in algorithm 3.1) is shown in Fig3. Compared with the transient response in Fig 3, the transient response in Fig 2 is better than that in Fig 3, which manifests that the feasible controller set method can improve the transient response of the switching system.

For further comparison, Fig 4 and Fig5 are the controller switching instants of algorithm 3.1 and the algorithm that does not adopted the feasible controller set method (no step 4 in algorithm 3.1) respectively. In Fig 4, at the first switching time, the observer $\mathrm{O}_{2}$ and controller $\Pi_{2}$ and $\Pi_{5}$ must not be the real controller and are excluded from the feasible controller set, Therefore the scope of the feasible controller set is reduced so that the controller converge more rapidly in Fig4 than in Fig 5 and the online estimation computation burden is reduced.

\section{CONCLUSIONS}

In this paper, a new class of adaptive switching mechanisms is presented. It is assumed that the uncertain plant to be controlled may be described by a model contained in a finite set of linear continuous parameter uncertain system models $\mathrm{P}$, and assumed that a finite set of robust controllers $\left\{\Pi_{i}\right\}, i=1,2, \ldots \ldots$ s, has been found so that for each plant $P_{i} \in P, i=1,2, \ldots . . s$, there exists one controller $\Pi_{i}$ , $i=1,2, \ldots . . s$, making the resultant closed-loop system stable, a switching control algorithm is proposed in this paper to select real controller out of controller set $\left\{\Pi_{i}\right\}$ with the property of stability of system. This approach is based on the feasible controller set which incorporates simultaneous falsification of a number of controllers, therefore, improves controller converge rate and reduces computation burden. The potential advantages of this approach include the finite convergence for switching, the simplicity of the stability analysis and the applicability to both continuous and discrete system. 


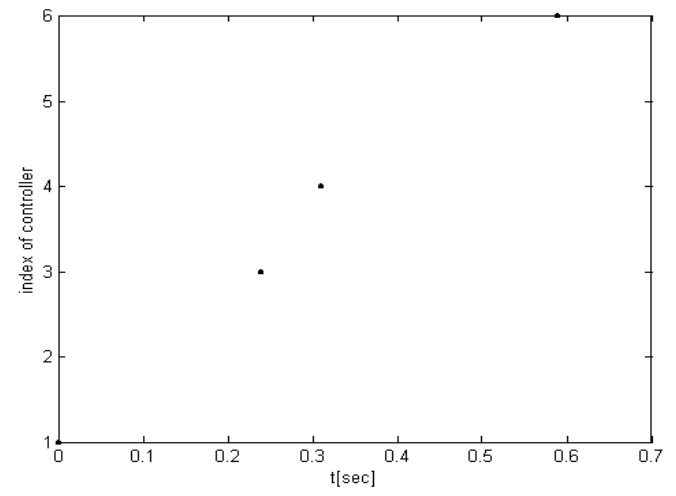

Figure 4 The controller switching instants of algorithm 3.1

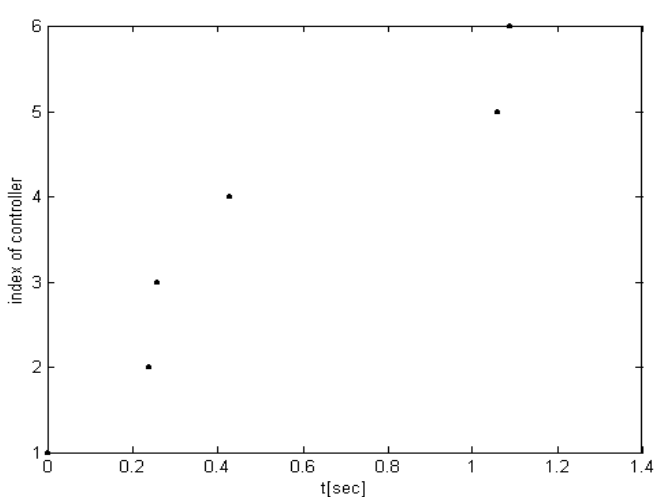

Figure 5 The controller switching instants that does not adapt feasible controller set method (no step4 in algorithm 3.1)

\section{References}

[1] J. Wang, "Switching control of a class of linear MIMO di screte systems," Control and Decision (in Chinese), vol 19, pp81-84, 2004.

[2] M.H. Chang and E.J. Dayision, "Adaptive switching control of LTI MIMO systems using a family of Controllers approach," Automatica, vol. .35, pp:453-465, 1999.

[3] Fu. Minyue and B.R.Barmish, "Adaptive Stabilization of linear systems via switching control," IEEE Trans Automat.Contr, vol. 31,pp 1097 1103,1986

[4] D.E. Miller and E.J. Dayision, "An adaptive controller which provides Lyapunov stability," IEEE Trans Automat.Contr, vol. 34, pp 599-609, 1989

[5] A.S. Morse. "Supervisory control of families of linear set-poin controllers," IEEE Trans Automat.Contr, vol. 41 pp 1413-1431, 1996

[6] S.R. Kukami. "Model and controller selection policies based on output errors," IEEE Trans Automat.Contr, vo1 41, pp1594-1604, 1996.

[7] H. Judith, S.R .Kukarmi and P.J. Ramadge. "Controller switching based on output prediction errors," IEEE Trans Automat.Contr, vol. 43, pp596$607,1998$.

[8] K.S.Narendra and J.Balakrishnan. "Adaptive control using multiple models,", IEEE Trans Automat.Contr, vol. 42,pp171-187, 1997.

[9] P.V. Zhivoglyadov, R. H. Middleton and Minyue Fu, "Localization based switching adaptive control for time-yarying discrete-time systems," IEEE Trans Automat.Contr, vol. 45,pp752-755,2000 